\title{
Sensitivity to time interval changes in speech and tone conditions
}

\author{
Simon Grondin • Nicolas Bisson • Caroline Gagnon
}

Published online: 23 November 2010

(C) Psychonomic Society, Inc. 2010

\begin{abstract}
The main goal of the present experiment was to verify whether sensitivity to time interval changes in speech is greater than sensitivity to time interval changes between tones. The 12-syllable sentences were delivered in French (the main language of the participants) or in a foreign language (Slovenian) that was totally unfamiliar to the participants. Two types of sub-sequences were induced within the series of stimuli (sub-sequences in sentences or in tone sequences). Discrimination, as revealed by the Weber fraction in each condition, was much better in the tone conditions than in the speech conditions. Nevertheless, discrimination was excellent in all conditions: Weber fractions below $2 \%$ with tones and around $4.5 \%$ in the speech conditions. Moreover, the study revealed that familiarity with a particular language does not affect performance and that inducing internal sub-sequences within tone conditions, as opposed to using a series of equal intervals, does not lead to better discrimination. The fact that discrimination is better with tones than with sentences indicates that the extensive training provided by speech does not lead to higher sensitivity to time interval variations than simple tones do. Instead, it seems to indicate that the different acoustical variations in speech reduce the capability to discriminate time interval variations.
\end{abstract}

Keywords Language $\cdot$ Speech $\cdot$ Interval discrimination

The environment, with its flow of events, is filled with occurrences of physical regularities (Jones, 1976; Jones \&

S. Grondin $(\bowtie) \cdot$ N. Bisson $\cdot$ C. Gagnon

École de psychologie, Université Laval,

2325 rue des Bibliothèques,

Québec City, QC, Canada G1V 0A6

e-mail: simon.grondin@psy.ulaval.ca
Boltz, 1989). Music and speech are often reported as fine examples of such occurrences of regularities. Periodic temporal structures are more obvious in music than in speech (Patel, 2008), but humans are very sensitive, for instance, to temporal variations during a conversation. There is now growing evidence that some fundamental connections exist between speech and music timing patterns (see Patel, 2008). Therefore, discriminating and producing rhythm or time intervals are basic abilities to detect and communicate messages efficiently.

As noted by Quené (2007), there are a number of reports on the capacity to detect tempo changes in music (Ellis, 1991) and with clicks (Madison, 2004). The just noticeable difference (JND) is about $4 \%$ with clicks and between 5 to $13 \%$ with music, depending on the base tempo under investigation. The capacity to discriminate tempo in music has been reported to be even better. For instance, when participants have to discriminate small tempo variations in a musical excerpt, sensitivity (Weber fraction) can be as low as $3 \%$, and even $1 \%$ for musicians (Grondin \& Laforest, 2004).

Despite its importance, the study of rhythm or of interval changes in the context of speech has been somewhat neglected. Only a few studies provide some estimations of the capability to discriminate tempo in speech. The earliest ones do not allow valid and reliable conclusions to be drawn regarding the capability of humans to discriminate tempo changes (Eefting \& Rietveld, 1989; Nooteboom \& Eefting, 1994; reported by Quené, 2007). However, Quené recently conducted a more systematic investigation of this question. In his experiments, participants were presented with a standard sentence and a sentence in which the tempo was accelerated or decelerated. Participants had to either indicate whether the sentences had the same tempo or not (Experiment 1) or determine which one was faster than the 
other (Experiment 2). Quené reported that the JND for tempo was about 5\%, with sensitivity for accelerations being 1 to $2 \%$ higher (lower JND) than sensitivity for decelerations. He also showed (Experiment 3) that, despite the compression and expansion of the sentences' temporal aspects, participants' judgments were really based on tempo rather than on other acoustic properties.

Although the experiments by Quené (2007) are the best conducted to date for estimating tempo capabilities in the context of speech with sentences, we cannot exclude the possibility that he slightly underestimated tempo discrimination levels. It is known that the choice of parameters can influence the estimated values of discrimination thresholds for time (Wearden \& Ferrera, 1995, 1996; but see Grondin, 2010). In Quené's study, the difference between the standard and comparison intervals varied from 5 to $20 \%$, - that is, only within a range beyond the estimated $5 \%$ value. Another relatively recent report on the same issue reveals a slightly smaller value for the Weber fraction for temporal discrimination with speech stimuli. In the experiment discussed in that report (see Experiment 2), Kato, Tsuzaki, and Sagisaka (2003) did not use sentences, but a series of four-syllable (mora) words, with each syllable consisting of a consonant and a vowel. The mean word duration was $713 \mathrm{~ms}$, and the modifications varied from 2.8 to $11.2 \%$. Kato et al. reported a Weber fraction of $3.5 \%$ when entire words were modified and one just above $5.1 \%$ when the vowel onsets were specifically modified. Like Quené, Kato et al. also observed better discrimination for accelerations than for decelerations.

There are also several reports on sensitivity for discriminating single time intervals in the auditory mode or for discriminating rhythms marked with tones. The JND is usually reported to be lower (higher sensitivity) with multiple intervals than with single intervals (Drake \& Botte, 1993; Grondin \& McAuley, 2009), and the JND values are reported to vary between 3 to $10 \%$ for audition, depending mainly on the range of duration under investigation and the method adopted for estimating JNDs. For very brief intervals (around $100 \mathrm{~ms}$ ), it has been reported that the JND of listeners with normal hearing is about 10 to $15 \%$ for filled intervals and for durational increments for vowels embedded in consonant-vowel-consonant words; however, it is closer to $30 \%$ for empty intervals (Bochner, Snell, \& MacKenzie, 1988). It is also known that listeners are more sensitive to changes in vowel duration than to changes in consonant duration (Huggins, 1972).

What is missing in the literature on rhythm or on time intervals changes is a direct comparison, with the same participants, of performance levels for sentences and tone conditions. One can reasonably posit that higher sensitivity to temporal variations would be favored by conditions such as the ones encountered in speech and probably in music.
Speech and music provide more natural stimulus variations than do the series of discrete tones usually employed in time psychophysics experiments, in which brief empty intervals are to be discriminated.

The only experiment that might resemble such a direct comparison is that of Lehiste (1977). Participants were asked to judge interstress intervals in sentences and in nonspeech conditions in which stress was replaced by clicks and speech by noise. Lehiste reported that detecting isochrony is easier with nonspeech than with speech stimuli. However, as noted by Patel (2008), it might not be the speech per se that impairs performance in the speech conditions but the presence of semantic meaning that would detract participants' attention from time. It is well known that attention is a critical factor in time performance (Brown, 2008; Macar, Grondin, \& Casini, 1994). To control for this potential explanation, Patel (2008) recommends adopting a condition in which the language is unfamiliar to participants.

The main goal of the present study was to compare potentially fine sensitivity to temporal variations in speech (sentences) with the sensitivity observed in comparable tone conditions, with the same participants. Small temporal variations were introduced in sentences and the method of constant stimuli was used. These temporal variations were smaller than those used by Quené (2007).

The experiment was conducted in French, with Frenchspeaking observers. The sentences were delivered not only in French, the main language of participants, but also in Slovenian, a foreign language the participants did not understand. With a foreign language, semantic meaning will not attract attentional resources (Patel, 2008). Moreover, the way speech is fragmented in a foreign language may be influenced by the native language (Cutler, 2000). During the experiment, we imposed certain grouping strategies that were similar in both languages. We used some temporal patterning, or grouping of syllables, that was expected to provide rhythm. Sentences were selected to favor a grouping of syllables often used in poetry for inducing rhythm. One sentence was divided rhythmically into 4 three-syllable fragments (3-3-3-3) and the other into 3 four-syllable fragments (4-4-4).

\section{Method}

\section{Participants}

Sixteen French-speaking Quebecers from Université Laval (eight women and eight men; mean age $=24.9$ ) volunteered to take part in the experiment. They had no knowledge of the foreign language chosen (Slovenian) and reported no hearing or language problems. They received \$48 CAN for their participation. 
Apparatus and stimuli

Each participant was seated in front of an IBM computer in a dimly lit room, and the stimuli were presented binaurally through headphones (Sennheiser HD 477) at approximately $70 \mathrm{~dB}$ SPL. After each trial, participants were asked to respond whether the second sentence (or sequence of tones) was "slower" or "faster" than the first one by pressing " 1 " or "3," respectively, on the computer keypad, and were instructed to avoid using an explicit count of numbers.

The sentences used in this experiment were recorded in a professional recording studio in Québec City. Cool Edit Pro version 2.0, now known as Abode Audition, was used for accelerating or decelerating each sentence in eight steps by increments of $1 \%$ (i.e., from -3.5 to $3.5 \%$ ). To that end, the duration of each sentence was respectively shortened (thus accelerating the speaker's voice) or lengthened (thus decelerating the speaker's voice). The time stretch tool provided in the software made it possible to change the speed of the sentences without affecting their pitch. ${ }^{1}$

Sentence conditions Two neutral declarative sentences were recorded in French and two in Slovenian. The Slovenian and French sentences were recorded by different speakers. In each language, one sentence was divided rhythmically into 4 three-syllable fragments (referred to as "3-3-3-3") and the other into 3 four-syllable fragments ("4-4-4").

In French, the 3-3-3-3 sentence was "Il avait demandé à François de l'aider," and the standard version lasted 2,054 ms. The 4-4-4 sentence was "Emmanuelle est repartie lundi matin," and the standard version lasted 2,093 ms. One male and one female native speaker of Québec French delivered each French sentence at a normal rate, for a total of four standard stimuli (sentences) in French. ${ }^{2}$

In Slovenian, the 3-3-3-3 sentence was "Predite, popoldne, do nase, bicise," and the standard version lasted 2,337 ms. The 4-4-4 sentence was "Solnce sije, kladvo bije, zgodnje ure," and the standard duration lasted

\footnotetext{
${ }^{1}$ We estimated the fundamental frequency with the Praat computer software and, for each sentence, it was almost the same in different length conditions (Boersma \& Weenink, 2010).

${ }^{2}$ There was a small difference between the male and the female original recordingdurations in each sentence condition. The largest difference occurred in the French 3-3-3-3 condition (1,999 vs. $2,109 \mathrm{~ms})$. To avoid any artefacts caused by differences between the gender's standard stimulus durations, the male and female recordings were either accelerated or decelerated slightly to obtain the same standard duration for both genders. This adjustment was applied to each language condition. For each sentence condition, the standard duration was the mean of the male and female recordings. Following these adjustments, these standard stimuli were then accelerated or decelerated to create all of the comparison stimuli. We conducted conventional physical measurements by recording the output sound and can confirm that the parameters described in the method did not change the output sound.
}

2,387 ms. One male and one female native speaker of Slovenian delivered each sentence at a normal rate, for a total of four standard stimuli (sentences) in Slovenian.

Tone conditions There were four tone (or control) conditions. In three of these four nonspeech conditions (tones), the intervals to be discriminated were marked by two $1-\mathrm{kHz}$ 20-ms auditory signals with a 5-ms rise and a 5-ms fall envelope (see Fig. 1a) marking a series of empty intervals. The fourth condition was a series of filled intervals. In each of the four conditions, the total duration from the onset of the first signal to the offset of the last signal was set so that it was very close to the total duration of the sentences.

In the two empty interval conditions, we tried to induce a rhythmic sequence that was close to the 4-4-4 and the 33-3-3 speech conditions. The 4-4-4 rhythmic pattern was generated by using progressively longer intervals before each fourth tone. All intervals were $175 \mathrm{~ms}$ long, except the one between the third and fourth tones $(190 \mathrm{~ms})$, the one between the seventh and eighth tones $(200 \mathrm{~ms})$, and the one between eleventh and twelfth tones $(210 \mathrm{~ms})$ (see Fig. 1b). The sequence lasted $2,240 \mathrm{~ms}$. To generate the 3-3-3-3 rhythmic pattern, all intervals lasted $170 \mathrm{~ms}$, except the one between the second and third tones $(180 \mathrm{~ms})$, the one between the fifth and the sixth tones $(203 \mathrm{~ms})$, the one between the eighth and ninth tones $(180 \mathrm{~ms})$, and the one between the eleventh and twelfth tone $(203 \mathrm{~ms}$ ) (see Fig. 1b). This sequence lasted 2,196 ms.

In a third control condition, a sequence of 11 equal empty intervals of $180 \mathrm{~ms}$ (between twelve 20-ms tones) was presented, for a total duration of 2,220 ms. Silent intervals between the tones in these three conditions were accelerated or decelerated, just as the sentences were (same percentage). In a fourth control condition, a sequence of 12 tones (filled intervals) separated by eleven 18-ms empty intervals was presented. The tones used for delimiting the intervals were $168-\mathrm{ms}, 1-\mathrm{kHz}$ continuous signals, with rise and fall times equal to $35 \mathrm{~ms}$ (Fig. 1b). The total duration of the sequence in this filled-interval condition was $2,214 \mathrm{~ms}$. To create the comparison intervals for this condition, the 98 -ms period (see Fig. 1a) of the 12 continuous signals was either accelerated or decelerated by the same percentage as the other tone conditions. In other words, regardless of the percentage of acceleration or deceleration, the duration of all 11 empty intervals in this filled-interval condition was always $18 \mathrm{~ms}$, and all 12 continuous signals always had $35-\mathrm{ms}$ rise and fall times.

\section{Procedure}

The experiment consisted of 12 experimental sessions (one session per condition, when the gender of the 
a

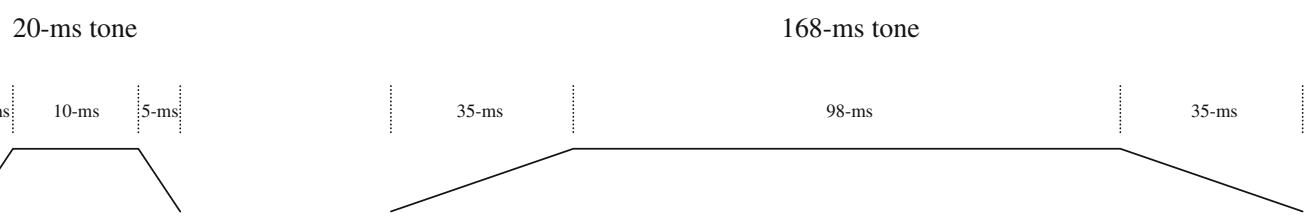

b

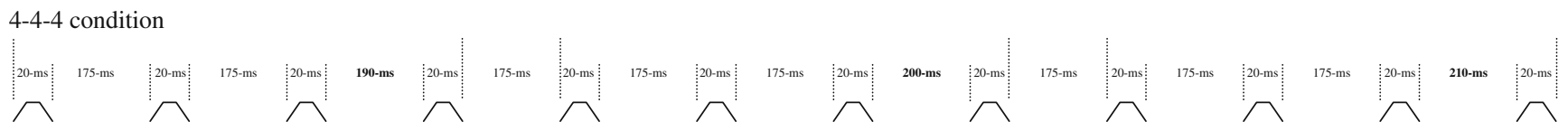

3-3-3-3 condition

Equal empty interval condition

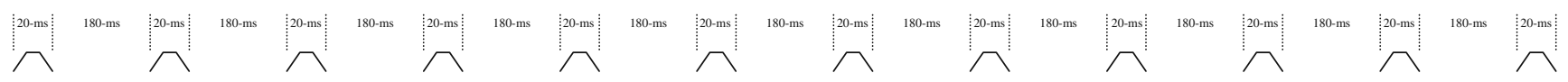

Equal filled interval condition

(168-ms

Fig. 1 a Auditory signals used in the tone conditions. The left illustration represents the 1-kHz 20-ms signals used in three (empty) conditions; the right illustration represents the $1-\mathrm{kHz} 168-\mathrm{ms}$ signals used in filled-interval condition. b Temporal structure of the four tone conditions

speaker in the "speech" condition is taken into consideration). Each session lasted approximately $30 \mathrm{~min}$. First, there were four control sessions in which the tone stimuli were presented; the order of presentation of the four conditions was counterbalanced between participants. The following eight sessions involved the presentation of the eight language stimuli, one per session, and the condition presentation order was also counterbalanced between participants. Each session was divided into two blocks of 72 trials. Participants had to take a period of rest between sessions, and were limited to a maximum of two sessions per day.

The stimuli were presented to participants using a two alternative forced choice paradigm. For each trial, the standard (St) was presented first, followed by one of the eight (i.e., from -3.5 to $3.5 \%$ ) electronically accelerated or decelerated comparison stimuli (Co). The choice of parameters was based on a pilot study. Each pair was presented nine times per block, for a total of 144 presented stimulus pairs per session. The presentation order of the eight Co intervals was randomized for each participant. There was 1,000 ms between the St and Co intervals, and the intertrial intervals lasted 2,000 ms. The procedure was the same for the four tone conditions and the eight language conditions.

\section{Data analysis}

In the speech condition, the data with the male and female voices were pooled. Pooling increased the number of judgments per data points on each function and improved the goodness-of-fit in each of the four speech conditions. For each participant and for each of the eight experimental conditions (four with tones and four with speech), an 8-point psychometric function was traced, plotting eight comparison intervals on the $x$-axis and the probability of responding "slower" (from -3.5 to $3.5 \%$ of the standard value) on the $y$-axis. The cumulative normal distribution was fitted to the resulting curves. Estimates of the standard deviation $(S D)$ on the psychometric function were determined as indicators of temporal sensitivity. Using $1 S D$ (or variance) is a common procedure for expressing temporal sensitivity (Grondin, 2008; Killeen \& Weiss, 1987). The $S D$ value was divided by the total length of the standard sentence or tone sequence, yielding the Weber fraction. Another dependent variable was the point of subjective equality (PSE). The PSE can be defined as the $x$ value corresponding to the 0.50 probability of "slower" responses on the $y$-axis. The total length of the sentences was subtracted from the PSE, yielding a constant error value. 


\section{Results}

Figure 2 reports the grouped psychometric function for each of the eight experimental conditions. The mean individual goodness-of-fit in each tone condition was highly satisfactory: All $R^{2}$ values were above .94 . In the speech conditions, the mean $R^{2}$ values were .872 in the French 4-4-4 and Slovenian 3-3-3-3 conditions, .81 in the Slovenian 4-4-4 condition, and .773 in the French 3-3-33 condition. In the last two cases, the lower mean value can mainly be explained by the very low values $(<.25)$ obtained by three participants (one in the Slovenian condition and two in the French condition).

Figure 3 shows the Weber fraction in each of the six conditions: 2 Rhythms (3-3-3-3; 4-4-4) × 3 Stimuli (tones, French, Slovenian) conditions. The $2 \times 3$ ANOVA with repeated measures indicates that the stimuli conditions were significantly different, $F(2,30)=$ $6.30, p<.01, \eta_{p}{ }^{2}=.30$. Post hoc analyses indicate that the tone condition led to better discrimination than did each of the speech conditions. The rhythm factor was only marginally significant, $F(1,15)=3.79, p=.07, \eta_{p}{ }^{2}=.20$, and the interaction effect was not significant.

Figure 4 shows the results in each of the four tone conditions. The one-way ANOVA with repeated measures indicates that some differences between tones conditions were marginally significant, $F(3,45)=2.74$, $p=.054, \eta_{p}{ }^{2}=.16$, with the lowest performances (highest Weber fractions) being observed in the rhythm conditions.

The same ANOVAs were conducted with the constant error as the dependent variable. The $2 \times 3$ analysis revealed no significant rhythm $(p=.15)$ or stimuli $(p=.09)$ effects and no interaction $(p=.16)$. Additionally, the one-way ANOVA on the four tone conditions revealed no significant effect.

\section{Discussion}

The present study provides answers to certain questions regarding relative levels of duration discrimination when intervals are embedded in a speech structure as opposed to when they are marked by tones. Moreover, the study makes it possible to compare duration discrimination of different temporal structures within different tone sequences and within sentences from familiar and foreign languages.

The answer with respect to relative sensitivity in speech and tone conditions is clear. Regardless of the temporal structures used with speech and tone stimuli and of whether or not participants understand sentence content, discrimination is better when sequences of simple tones are presented than when speech stimuli are presented. In other words, keeping nontemporal features simple and constant, as is the case with tones but not with speech stimuli, facilitates temporal discrimination. Indeed, the lowest Weber fraction observed in this study in the best speech condition was almost twice as high as the highest Weber fraction in the tone conditions. Moreover, using a foreign language led to only small benefits (4.2\% Weber fraction) as compared with using a familiar language (4.7\%), when the difference was compared to that observed using tones. Therefore, the difference between the speech and nonspeech conditions in Lehiste (1977)'s experiment can probably not be explained, contrary to Patel's (2008) hypothesis, by the use of a familiar language. Knowing the semantic meaning of a language is expected to solicit attentional resources that are therefore not available for discriminating rhythms. In fact, the predicted difference was not observed.

In the present study, discrimination levels in speech conditions were higher (about 4.5\% Weber fraction overall) than the level reported by Quené (2007, 5\% Weber fraction). It cannot be excluded that this small difference is due to a benefit obtained with the rhythmic structure used in the present study. However, this explanation seems unlikely when one considers that the best performance in the tone conditions was obtained without sub-sequences. On the other hand, the difference in performance levels $(4.5 \%$ vs. $5 \%)$ between our study and Quené's can be partly explained by the different methodologies used. For example, the parameters employed in our study for estimating thresholds were not the same as those in Quené's. Moreover, Quené's study used a large sample of sentences, whereas our experiment presented the same sentences multiple times.

Although the overall interval discrimination in speech contexts was excellent irrespective of the rhythmic patterns induced by the temporal structures (3-3-3-3 or 4-4-4), the performance level was lower than that $3.5 \%$ Weber fraction) reported by Kato et al. (2003). Note, however, that Kato et al.'s experiment was conducted in Japanese and, most importantly, contained only compressions and expansions of words (all four-syllable words) and not sentences. Indeed, the level of discrimination observed by Kato et al. was close to the one obtained by nonmusician participants for temporal discrimination in musical excerpts (Grondin \& Laforest, 2004). On the other hand, when Kato et al. made a change to the temporal structure of the vowel onsets rather than to the global temporal structure, the Weber fraction $(5.1 \%)$ was like the one reported by Quené (2007).

An important feature of the present study is that it also allowed the comparison of temporal structures when tones alone were used. The Weber fractions reported here are all very low. It is often reported that the Weber fraction for 
Fig. 2 Psychometric function (grouped data) in each experimental condition
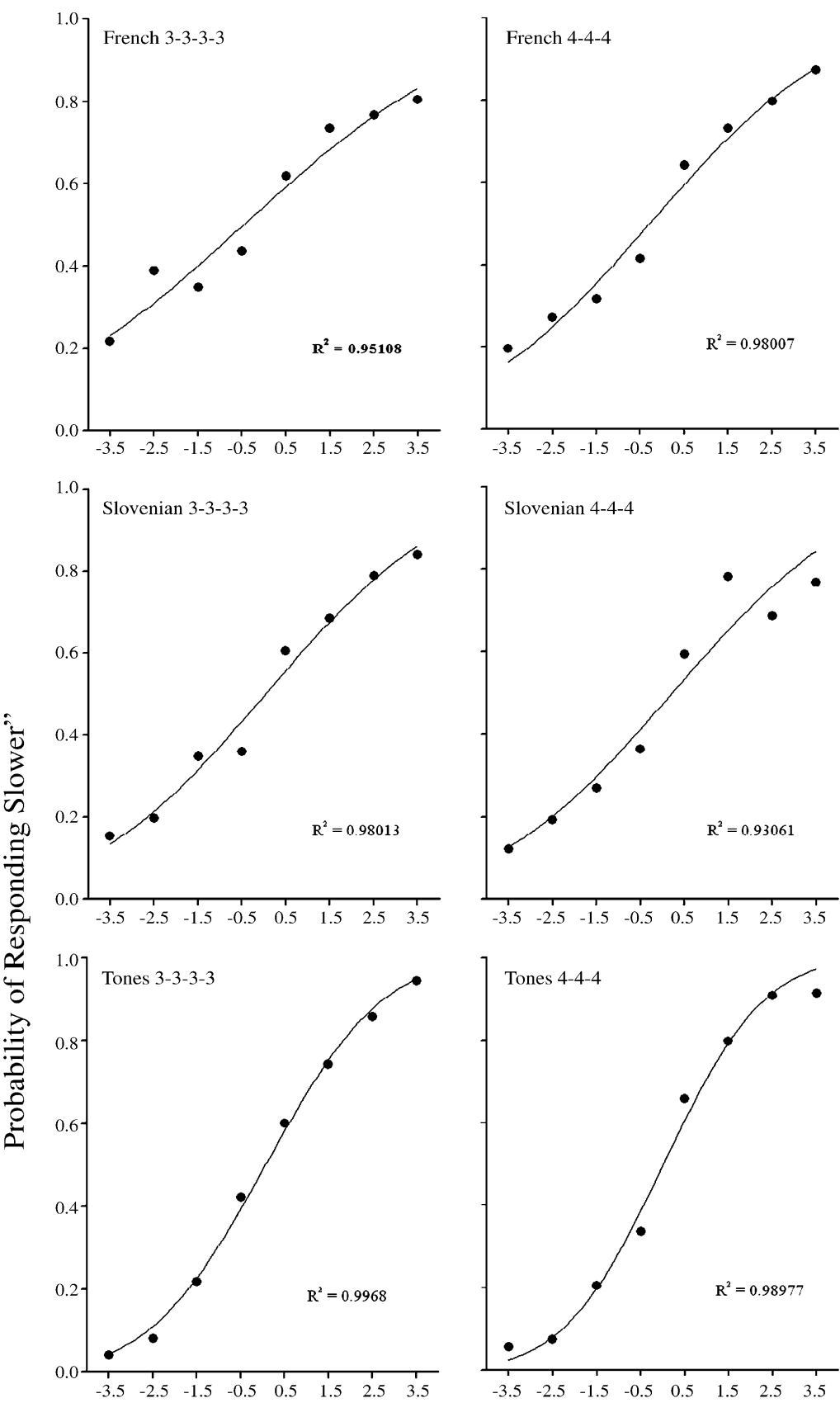

Slovenian 4-4-4
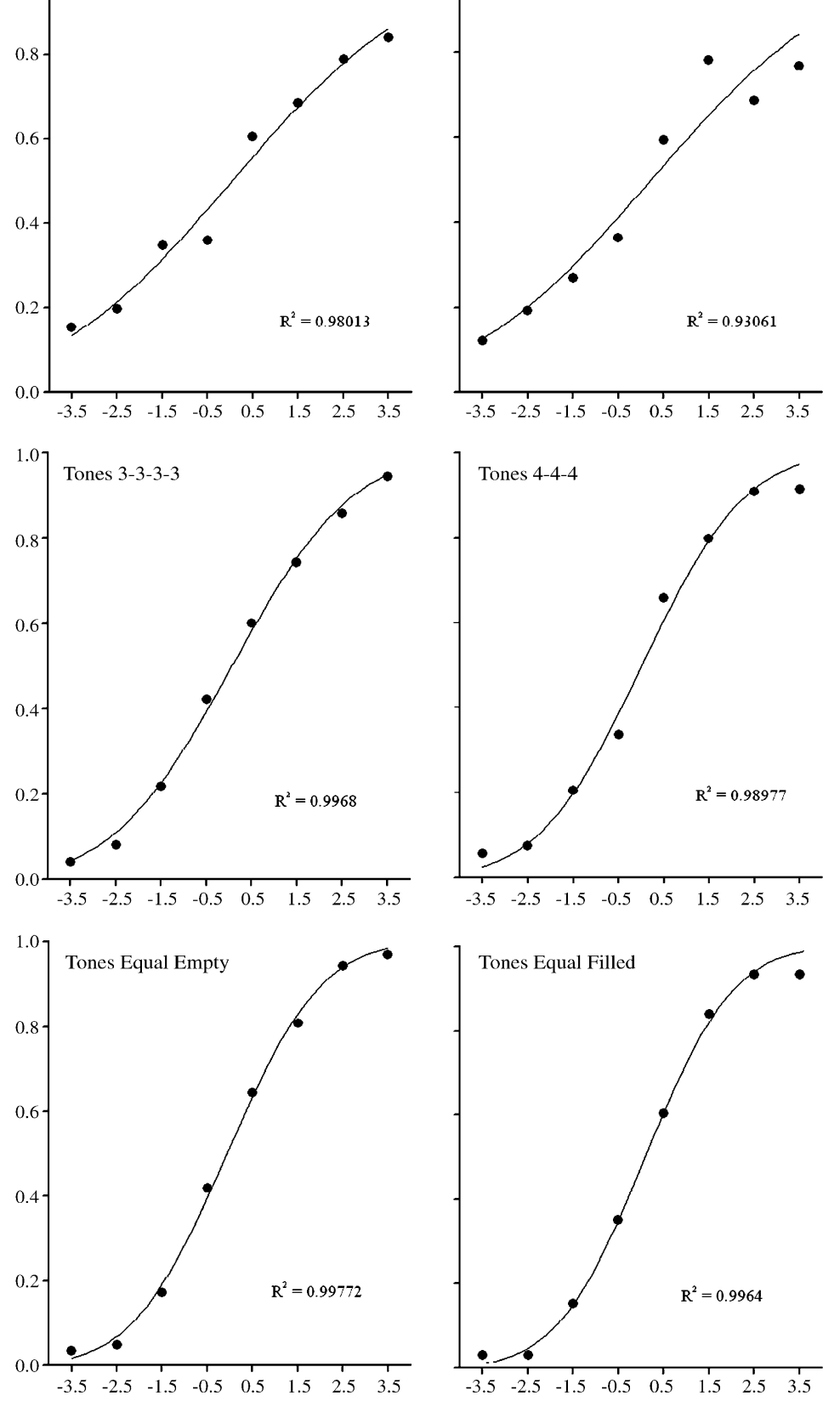

Comparison Interval (\%) 


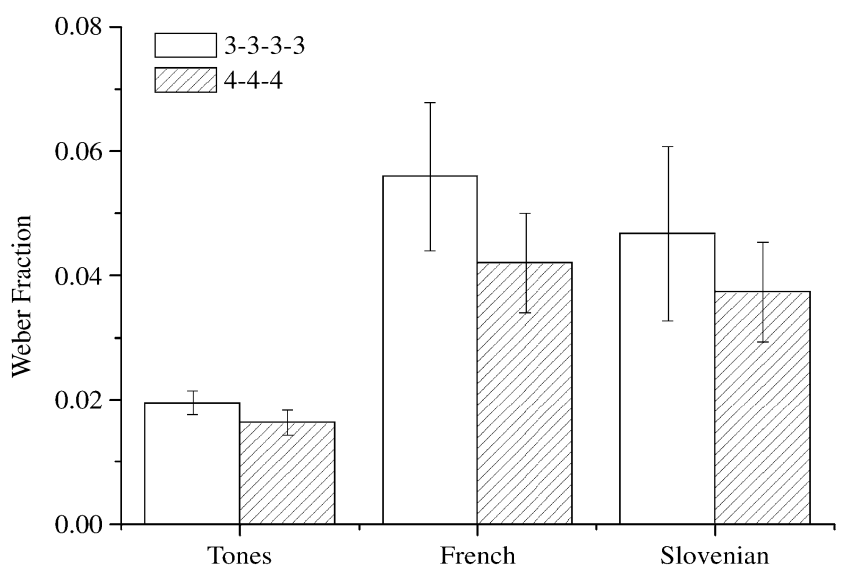

Fig. 3 Mean Weber Fraction in each rhythm (3-3-3-3; 4-4-4) condition as a function of stimulus condition (tones, French, Slovenian). Bars represent $S E$ s

temporal discrimination is about 5 to $10 \%$ in the auditory mode (Ehrlé \& Samson, 2005; Friberg \& Sundberg, 1995) and only slightly higher when intervals are very brief (<100 ms; Hirsh, Monahan, Grant, \& Singh, 1990). Indeed, discrimination levels are known to depend on the number of intervals presented (Grondin \& McAuley, 2009; McAuley \& Miller, 2007; Miller \& McAuley, 2005). Multiple intervals were presented in our study, leading to Weber fraction values that were much lower (close to $1.5 \%$ ) than those usually reported for tempo discrimination. The values were consistent with the data reported by Drake and Botte (1993). Such low Weber fractions may reveal that the auditory system is particularly sensitive to temporal variations in the temporal range that is in the vicinity of intervals encountered in speech. However, the low Weber fractions of Drake and Botte also apply to intervals of up to about $600 \mathrm{~ms}$, which are much longer than the intervals usually embedded within speech.

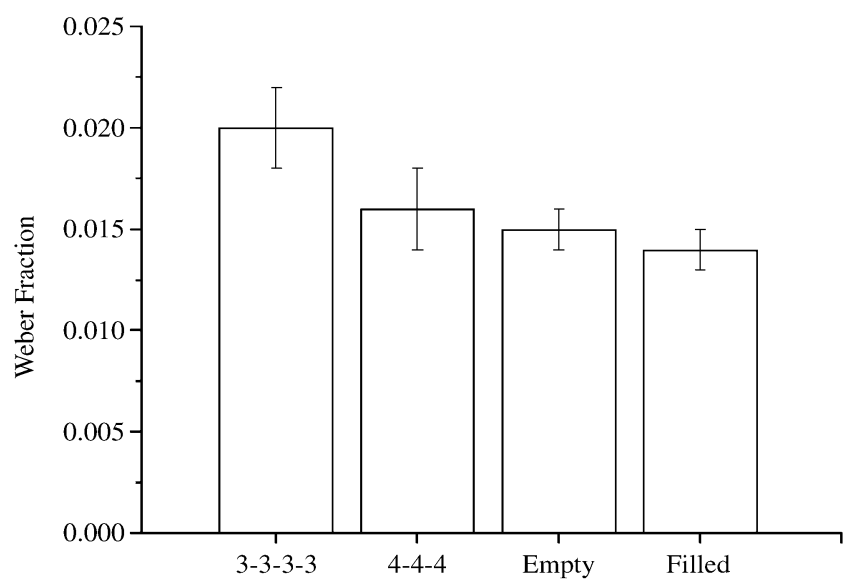

Fig. 4 Mean Weber Fraction in each of the four tone conditions (3-33-3, 4-4-4, empty, and filled "intervals"). Bars are SEs
The present study also revealed that no gain can be expected from including rhythmic patterns in sequences of tones. Observers were not able to rely on a second level of rhythm for increasing discrimination levels. The benefit in tone conditions was probably dependent on multiple repetitions of the same intervals and, therefore, on consolidating the representation in memory (Drake \& Botte, 1993). In addition, the fact that discrimination in the filled interval condition was at least as good as it was with empty intervals indicates that the high performance levels were linked to a repetition effect rather than to some rhythmic benefit. In brief, the study showed that no benefit can be expected from the induction of rhythmic patterns within a series of tones and that using a long series of successive filled $(1.40 \%)$ and empty $(1.51 \%)$ intervals leads to approximately the same level of discrimination.

Finally, there were no significant differences between the experimental conditions for constant error. Indeed, there was not much difference between the highest $(+2.7 \mathrm{~ms})$ and the lowest $(-1.3 \mathrm{~ms})$ values in the tone conditions. With sentences, the constant error varied from $+8.2 \mathrm{~ms}$ in the Slovenian 4-4-4 condition to $-15.2 \mathrm{~ms}$ in the French 3-3-3-3 condition. The other two speech conditions also led to negative constant errors $(-10.5$ and -6.1 in the other French and Slovenian conditions, respectively). A positive value, as in the Slovenian 4-4-4 condition, indicates that participants tended to respond "faster" more often and, therefore, to give more good responses when the comparison intervals were accelerated. This seems to be consistent with the better discrimination for accelerations than for decelerations reported by Kato et al. (2003) and by Quené (2007; see also Pouliot \& Grondin, 2005, for a comparable result with musical excerpts). However, the present results were not significant, and it is difficult to understand why a positive value was obtained only in the Slovenian 4-4-4 condition and not, for instance, in the other Slovenian condition or in the other 4-4-4 condition.

\section{Limitations}

The French and Slovenian sentences used in the present study were not of exactly the same length. It cannot be excluded that this difference influenced the Weber fraction estimates. However, the best performance (lower Weber fraction) out of four was obtained in Slovenian (i.e., in the condition with with the longest sentences), and the second best performance was obtained in French (i.e., in the condition with the briefest sentences). Moreover, some sentences were briefer and others longer than the tone sequences. In both cases, discrimination was much better with the tones. In brief, we do not believe that the duration of sentences and tone sequences was a factor in 
the different sensitivity levels observed with tones versus with sentences. ${ }^{3}$

Since different sentences were used for comparing the familiar versus foreign language issue, it is not possible to draw a definite conclusion about this matter. A betweensubjects design, with groups of participants from different countries, would make it possible to test this familiar versus foreign issue with the same set of sentences. Although the use of different sentences might be viewed as a weakness of the present study, the experiment remains valid because of its within-subjects design.

It is also possible that comparing speech and tone conditions when no syllable grouping is used would lead to exactly the same results. The results in the tone conditions in this experiment indicate that grouping tends to decrease performance; however, no such grouping in sentences was used in the experiment by Quené (2007), and the Weber fractions were no smaller than the one reported here.

Finally, given the experimental design used in the present study, it cannot be totally excluded that the superiority of tone conditions over speech conditions was caused by the accumulated fatigue in the speech conditions (Sessions 5-12). However, there were no signs of fatigue in the performance since the Weber fraction in Session 12 was equivalent to that in Session 6. Indeed, an ANOVA revealed no significant difference between the eight sessions with the speech conditions. Additionally, the Weber fraction in the first session with speech stimuli (Session 5) was more than doubled when compared with the last session with tones (Session 4). Moreover, as indicated in the Method section, participants had to take a period of rest between sessions and were limited to a maximum of two sessions per day.

\section{Conclusion}

In his volume on music, speech, and the brain, Patel (2008, p. 144) reports the data obtained by Drake and Botte for tempo

\footnotetext{
${ }^{3}$ Additionally, it is very unlikely that the temporal judgments were based on the entire stimulus duration (i.e., on intervals $>2$ s). The Weber fractions are simply too low ( $<2 \%$ with tones) for one to believe that discrimination was based on very long intervals ( $>2 \mathrm{~s})$. It is known, for instance, that Weber fractions are likely higher at $1 \mathrm{~s}$ than at $.2 \mathrm{~s}$ (Grondin, 2010; it is close to $200 \mathrm{~ms}$ in the present study), and are even higher for rhythm at $1.5 \mathrm{~s}$ than at $1 \mathrm{~s}$ (Drake \& Botte, 1993). Maybe even more importantly, with intervals as long as $2 \mathrm{~s}$, the only way to keep the Weber fraction as low as what is observed in the present tone conditions would be to have participants adopting an explicit counting strategy (Grondin, Meilleur-Wells, \& Lachance, 1999), which was not the case in the present study. Indeed, as indicated in the Method section, participants were asked to avoid explicit counting, although it would have been very unlikely that one would have adopted spontaneously such a strategy given the nature of the task (i.e., a task in which multiple stimuli, and not an empty duration, were presented).
}

discrimination and wonders what the threshold would be if speech sounds were used. The present article provides a clear response to this simple but important question. Discriminating time interval changes is easier with sequences of simple tones than with speech stimuli, regardless of whether familiar or foreign languages are used.

Acknowledgements This research was made possible by a research grant awarded to S.G. by the Natural Sciences and Engineering Research Council of Canada (NSERC), and an NSERC Summer Scholarship awarded to C.G. and to N.B. This work was presented at the Rhythm Perception and Production Workshop held in Lille, France in July 2009, and at the APCAM meeting held in Boston in November 2009. We would like to thank Lisa Hawke for helping us to conduct the experiment, Tsuyoshi Kuroda for his fine technical assistance, and Hugo Quené and one anonymous reviewer for their comments and suggestions on a previous version of this article. Correspondence should be addressed to Simon Grondin, École de psychologie, 2325 rue des Bibliothèques, Université Laval, Québec, Qué., Canada G1V 0A6 (simon.grondin@psy.ulaval.ca)

\section{References}

Bochner, J. H., Snell, K. B., \& MacKenzie, D. J. (1988). Duration discrimination of speech and tonal complex stimuli by normally hearing and hearing-impaired listeners. The Journal of the Acoustical Society of America, 84, 493-500.

Boersma, P., \& Weenink, D. (2010). Praat (Version 5.1.30) [Computer software]. Retrieved from http://www.fon.hum.uva.nl/praat/

Brown, S. W. (2008). Time and attention: Review of the literature. In S. Grondin (Ed.), Psychology of time (pp. 111-138). Bingley, England: Emerald Group.

Cutler, A. (2000). Listening to a second language through the ears of a first. Interpreting, 5, 1-23. doi:10.1075/intp. 5.1.02cut

Drake, C., \& Botte, M.-C. (1993). Tempo sensitivity in auditory sequences: Evidence for a multiple-look model. Perception \& Psychophysics, 54, 277-286.

Eefting, W., \& Nooteboom, S. G. (1993). Accentuation, information value and word duration: Effects on speech production, naturalness and sentence processing. In V. J. Van Heuven \& L. C. Pols (Eds.), Analysis and synthesis of speech: Strategic research towards high quality text-to-speech generation (pp. 225-240). Berlin: Mouton de Gruyter.

Eefting, W., \& Rietveld, A. (1989). Just noticeable differences of articulation rate at sentence level. Speech Communication, 8, 355361. doi:10.1016/0167-6393(89)90017-4

Ehrlé, N., \& Samson, S. (2005). Auditory discrimination of anisochrony: Influence of the tempo and musical backgrounds of listeners. Brain and Cognition, 58, 133-147. doi:10.1016/j.bandc.2004.09.014

Ellis, M. C. (1991). Thresholds for detecting tempo change. Psychology of Music, 19, 164-169. doi:10.1177/0305735691192007

Friberg, A., \& Sundberg, J. (1995). Time discrimination in a monotonic, isochronic sequence. The Journal of the Acoustical Society of America, 98, 2524-2531. doi:10.1121/1.413218

Grondin, S. (2008). Methods for studying psychological time. In S. Grondin (Ed.), Psychology of time (pp. 51-74). Bingley: Emerald Group.

Grondin, S. (2010). Unequal Weber fractions for the categorization of brief temporal intervals. Attention, Perception \& Psychophysics, 72, 1422-1430. doi:10.3758/APP.72.5.1422

Grondin, S., \& Laforest, M. (2004). Discriminating slow tempo variations in a musical context. Acoustical Science \& Technology, $25,159-162$. 
Grondin, S., \& McAuley, J. D. (2009). Duration discrimination in crossmodal sequences. Perception, 38, 1542-1559. doi:10.1068/ p6359

Grondin, S., Meilleur-Wells, G., \& Lachance, R. (1999). When to start explicit counting in a time-intervals discrimination task: A critical point in the timing process by humans. Journal of Experimental Psychology: Human Perception and Performance, 25, 993-1004. doi:10.1037/0096-1523.25.4.993

Hirsh, I. J., Monahan, C. B., Grant, K. W., \& Singh, P. G. (1990). Studies in auditory timing: 1. Simple patterns. Perception \& Psychophysics, 47, 215-226.

Huggins, A. W. F. (1972). Just noticeable differences for segment duration in natural speech. The Journal of the Acoustical Society of America, 51, 1270-1278.

Jones, M. R. (1976). Time, our lost dimension: Toward a new theory of perception, attention, and memory. Psychological Review, 83, 323-355. doi:10.1037/0033-295X.83.5.323

Jones, M. R., \& Boltz, M. (1989). Dynamic attending and responses to time. Psychological Review, 96, 459-491. doi:10.1037/0033295X.96.3.459

Kato, H., Tsuzaki, M., \& Sagisaka, Y. (2003). Functional differences between vowel onsets and offsets in temporal perception of speech: Local-change detection and speaking-rate discrimination. The Journal of the Acoustical Society of America, 113, 3379-3389.

Killeen, P. R., \& Weiss, N. A. (1987). Optimal timing and the Weber function. Psychological Review, 94, 455-468. doi:10.1037/0033295X.107.3.430

Lehiste, I. (1977). Isochrony reconsidered. Journal of Phonetics, 5, $253-263$
Macar, F., Grondin, S., \& Casini, L. (1994). Controlled attention sharing influences time estimation. Memory \& Cognition, 22, 673-686.

Madison, G. (2004). Detection of linear temporal drift in sound sequences: Empirical data and modelling principles. Acta Psychologica, 117, 95-118. doi:10.1016/j.actpsy.2004.05.004

McAuley, J. D., \& Miller, N. (2007). Picking up the pace: Effects of global temporal context on sensitivity to the tempo of auditory sequences. Perception \& Psychophysics, 69, 709-718.

Miller, N., \& McAuley, J. D. (2005). Tempo sensitivity in isochronous tone sequences: The multiple-look model revisited. Perception \& Psychophysics, 67, 1150-1160.

Nooteboom, S., \& Eefting, W. (1994). Evidence for the adaptive nature of speech on the phrase level and below. Phonetica, 51, 92-98. doi:10.1159/000261961

Patel, A. D. (2008). Music, language, and the brain. Oxford: University press.

Pouliot, M., \& Grondin, S. (2005). A response-time approach for estimating sensitivity to auditory tempo changes. Music Perception, 22, 389-399. doi:10.1525/mp.2005.22.3.389

Quené, H. (2007). On the just noticeable difference for tempo in speech. Journal of Phonetics, 35, 353-362. doi:10.1016/j.wocn. 2006.09.001

Wearden, J. H., \& Ferrara, A. (1995). Stimulus spacing effects in temporal bisection by humans. The Quarterly Journal of Experimental Psychology, 48, 289-310. doi:10.1080/14640749508401454

Wearden, J. H., \& Ferrara, A. (1996). Stimulus range effects in temporal bisection by humans. The Quarterly Journal of Experimental Psychology, 49, 24-44. doi:10.1080/713932615 